\title{
Development and Use of Machine-Learnt Algebraic Reynolds Stress Models for Enhanced Prediction of Wake Mixing in LPTs
}

\author{
H.D. Akolekar; J. Weatheritt, \\ N. Hutchins, R.D. Sandberg \\ Department of Mechanical Engineering \\ University of Melbourne \\ Parkville, VIC 3010, Australia \\ Email: hakolekar@student.unimelb.edu.au
}

\author{
G. Laskowski \\ General Electric Aviation \\ Lynn, MA 01905, USA \\ laskowsk@ge.com
}

\author{
V. Michelassi \\ Baker Hughes, a GE Company \\ Florence 50127, Italy \\ vittorio.michelassi@bhge.com
}

\begin{abstract}
Non-linear turbulence closures were developed that improve the prediction accuracy of wake mixing in low-pressure turbine (LPT) flows. First, RANS calculations using five linear turbulence closures were performed for the T106A LPT profile at isentropic exit Reynolds numbers 60,000 and 100,000. None of these RANS models were able to accurately reproduce wake loss profiles, a crucial parameter in LPT design, from direct numerical simulation (DNS) reference data. However, the recently proposed $k \overline{v_{2}} \omega$ transition model was found to produce the best agreement with DNS data in terms of blade loading and boundary layer behavior and thus was selected as baseline model for turbulence closure development. Analysis of the DNS data revealed that the linear stress-strain coupling constitutes one of the main model form errors. Hence, a gene-expression programming (GEP) based machine-learning technique was applied to the high-fidelity DNS data to train non-linear explicit algebraic Reynolds stress models (EARSM), using different training regions. The trained models were first assessed in an a priori sense (without running any CFD) and showed much improved alignment of the trained models in the region of training. Additional RANS calculations were then performed using the trained models. Importantly, to assess their robustness, the trained models were tested both on the cases they were trained for and on testing, i.e. previously not seen, cases with different flow features. The developed models improved prediction of the Reynolds stress, TKE production, wake-loss profiles and wake maturity, across all cases.
\end{abstract}

\section{INTRODUCTION}

Designing the blades of low-pressure turbines (LPTs) is a challenging task due to the large variation in the flow physics as the aircraft takes off and enters cruise conditions. At cruise conditions the turbine inlet chord Reynolds number is 2-5 times less than at sea-level take-off conditions which can cause the flow regimes to change from turbulent to transitional [1]. The prediction of such flows in LPTs is challenging as the inflow disturbance level is quite high and the blade boundary layers are subject to strong pressure gradients. The LPT has favorable pressure gradients up to mid-chord on the suction side and the flow switches over to an adverse- pressure gradient on the latter half of the suction side [2].

The flow over LPTs, at low isentropic exit Reynolds num-

\footnotetext{
*Address all correspondence to this author.
}

bers $(50,000-150,000)$, is characterized by laminar boundary layers which undergo separation-induced transition that leads to the formation of turbulent wakes [3]. Accurate prediction of the separation onset region and reattachment, turbulent-wake mixing and wake loss-profiles (which are crucial for engine blade designers) is a great challenge for existing Reynolds Averaged Navier-Stokes (RANS) based turbulence models. While some RANS-based turbulence models with transitional capabilities offer good prediction of the boundary layer states on the blade, and hence achieve reasonably accurate loading predictions, there still remains plenty of scope for improvement of wake mixing and wake-loss profile prediction [4,5]. It is crucial to bring about improvements in the RANS modeling for wake mixing in LPTs, as Praisner et. al [6], who conducted a study on engine performance impacts due to wake mixing in LPTs, suggested that losses due to wake-mixing can contribute up to 1.5 percentage point of lost efficiency in LPTs.

One of the reasons that RANS closures fail at predicting the turbulent mixing with the required accuracy is the use of the Boussinesq approximation $[7,8]$ for the stress-strain relationship. A computationally efficient approach to address the deficiencies of the Boussinesq approximation are explicit algebraic Reynolds stress models (EARSM) [9-11], which target the Reynolds stress anisotropy; and their development is the main focus of this work. Instead of using a conventional analytical approach, this work focuses on machine-learning based techniques to generate nonlinear explicit algebraic stress models (EARSM)s.

Data-driven techniques have been applied to turbulence modeling in numerous contexts. Wang et. al [12] proposed a high-fidelity data-driven, physics informed machine learning approach to improve discrepancies in RANS modeled Reynolds stresses. They developed models on particular training regions and tested them on different geometries for each of the two cases - square duct and periodic hills, and brought about improvements in the Reynolds stress distribution. They, however, were unable to propagate the improvement of the Reynolds stresses through the RANS equations to the mean velocity field. Duraisamy et. al [13] modified the SA model using neural networks and inverse modeling. However, this method is quite expensive as there is an optimization parameter for each of the grid points. The improved model was tested on the same case as it was developed. Therefore, the robustness or the ability of this model to generalize different flows is yet to be demonstrated. Tracey et. al [14] used a neural network to train an SA turbulence model and explored the potential of these networks to reproduce results over 
different simple flow configurations. This work depicted that the trained models can be used on flows different from the ones they were trained on.

Ferreira [15] introduced gene expression programming (GEP), which was a new adaptive algorithm for solving regression based problems. Weatheritt \& Sandberg [16] developed the GEP for turbulence modeling, which generates new EARSM closures. The algorithm not only improved the Reynolds stress prediction but also was shown to improve the mean flow prediction for a few canonical cases [17]. GEP is relatively inexpensive as compared to other machine learning techniques used for previous turbulence model studies. The EARSMs developed using GEP also have the added advantage that they do not require any high-fidelity data or supporting machine-learning based framework for their implementation into and use in CFD codes. GEP was used to conduct a priori analysis for a high pressure turbine vane, which showed significant improvement as compared to the baseline cases [18]. However, no a posteriori analysis (RANS calculation) was conducted in that work.

In this study, an extensive analysis of five RANS-based turbulence and transition models has been conducted on a LPT blade for isentropic exit Reynolds numbers of 60,000 and 100,000 , with the aim at identifying the potential for model improvement. The $k \overline{v_{2}} \omega$ transition model [19] was, to our knowledge, used for the first time for flow over LPTs. Further, EARSMs have been developed using the $k \overline{v_{2}} \omega$ transition model as the baseline turbulence transport equations, with the aim to improve the predictive accuracy of the overall turbulent wake mixing and wake-loss profiles. The GEP approach [16] was applied to high-fidelity data obtained from previously conducted direct numerical simulations (DNS) [20]. The developed EARSMs were tested both in an a priori (without running any CFD) manner, and have been executed successfully as RANS calculations for LPTs.

\section{RANS MODELING}

The most prevalent RANS models use the Boussinesq approximation to relate stress to strain which can be broken up into the sum of isotropic and anisotropic contributions

$$
\tau_{i j}=\underbrace{\frac{2}{3} \rho k \delta_{i j}}_{\text {isotropic }}-\underbrace{2 \mu_{t}\left(S_{i j}-\frac{1}{3} \frac{\partial u_{k}}{\partial x_{k}} \delta_{i j}\right)}_{\text {anisotropic }} .
$$

Beginning from the weak equilibrium hypothesis [9], a class of turbulence closures, known as EARSMs [21] were developed to express a tensor basis $V_{i j}^{k}$ and scalar invariants $I_{k}$ for $a_{i j}$; which will address the shortcomings of the linear stress-strain relation. Equation (2) defines $a_{i j}$, which is normalized by $2 \rho k$, for both Boussinesq and EARSM approximations

$$
\begin{aligned}
a_{i j} & \equiv \frac{\tau_{i j}}{2 \rho k}-\frac{1}{3} \delta_{i j} \quad(\text { Boussines } q) \\
& =a_{i j}\left(V_{i j}^{1}, V_{i j}^{2}, V_{i j}^{3}, \ldots, I_{1}, I_{2}, \ldots\right)(\text { EARSM }) .
\end{aligned}
$$

This tensor basis and set of scalar invariants are functions of the non-dimensional strain and rotation rate tensors, denoted by $s_{i j}=$ $\tau S_{i j}^{\prime}$ and $w_{i j}=\tau \Omega_{i j}$; where $\tau$ is the time scale denoted by $1 / \omega$ For two-dimensional flow, the basis and invariants are:

$$
\begin{aligned}
& V_{i j}^{1}=s_{i j}, V_{i j}^{2}=s_{i k} w_{k j}-w_{i k} s_{k j}, \\
& V_{i j}^{3}=s_{i k} s_{k j}-\frac{1}{3} \delta_{i j} s_{m n} s_{n m}, \\
& I_{1}=s_{m n} s_{n m}, I_{2}=w_{m n} w_{n m} .
\end{aligned}
$$

\section{FLOW CONFIGURATION}

Figure 1 depicts the T106A LPT blade geometry and computational domain (used for both the RANS and DNS) along with the inflow and outflow angles, inlet and outlet boundaries, and upstream and downstream measurement locations. The simu-

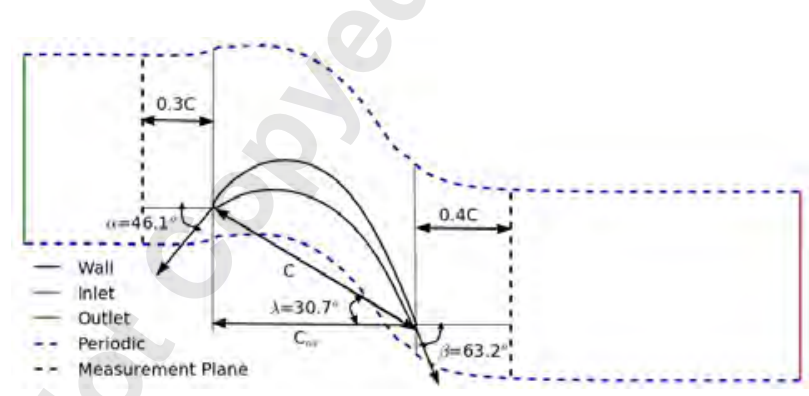

Fig. 1: Sketch of the T106A blade geometry and domain boundaries

lation employs a pitch-wise periodic boundary condition. The chord length $(C)$ is $0.1 \mathrm{~m}$, the pitch to chord ratio 0.799 and the inflow angle is $46.1^{\circ}$, which represents design conditions. The simulations in this study are 2D-URANS with compressible flow and have steady inflow conditions, i.e no incoming wakes and have been conducted in OpenFOAM with bounded second-order implicit schemes for spatial and temporal discretization. The grid used has 270,000 points in plane and 860 points around the blade, and is the same one used for the DNS studies, for which a thorough grid refinement study was performed. Using the same grid as the DNS [22] ensures that any errors in the RANS calculations are only due to the turbulence models and not due to the grid. There are 25-30 points across the boundary layer and $y^{+}<0.4$ for the first-off-the-wall points on both sides of the blade. The DNS results [20], used for comparing the RANS against and for EARSM development have been generated from a highlyoptimized code HiPSTAR (High Performance Solver for Turbulence and Aeroacoustics Research) [22] and have been shown to compare very well with experiments [23]. The wake-loss and wall-shear stress profiles of the DNS are in excellent agreement with the experiments at different inlet turbulence intensities and isentropic exit Reynold numbers $\left(\operatorname{Re}_{2 i s}=60,000 \& 100,000\right)$ based on the real chord. Table 1 depicts the operating conditions for both the RANS and DNS cases. Analyses for two isentropic exit Reynolds numbers have been conducted.

\section{URANS FOR LPTS}

In order to assess the effectiveness of RANS-based turbulence and transition models to predict the blade loading, boundary-layer flow and turbulent wake-mixing for LPTs, five 
Table 1: Operating conditions for RANS \& DNS

\begin{tabular}{lc}
\hline Parameter & Value \\
\hline Exit isentropic Reynolds Number $\left(\mathrm{Re}_{2 i s}\right)$ & $60,000 \& 100,000$ \\
Cascade Exit Mach number $\left(M_{2}\right)$ & 0.404 \\
Cascade Inlet Mach number $\left(M_{1}\right)$ & 0.24 \\
Inlet Turbulence Intensity & $4 \%$ \\
Inlet Length scale & $5 \% \mathrm{C}_{a x}$ \\
\hline
\end{tabular}

RANS models have been used. The $k \omega$-SST model [24] and the SA [25] model are the turbulence models used. One of the transition models used is the $\gamma-R e_{\theta}$ model of Langtry \& Menter [26] which models the intermittency and transition momentum thickness Reynolds number. The LKE model [27] determines the laminar, transition and turbulent flow regimes with the help of a concept known as laminar kinetic energy. LKE or laminar fluctuations are the fluctuations created by Tollmien-Schlichting waves in the pre-transitional boundary-layers [28]. The $k \overline{v_{2}} \omega$ model [19] describes the transition process with the $\overline{v_{2}}$ variable. In the pre-transitional boundary layer $\overline{v_{2}}$ corresponds to energy of the fluctuating velocity components normal to the streamlines. In fully turbulent flow, it corresponds to the energy of fully turbulent fluctuations [29]. At the inlet, $k$ and $\overline{v_{2}}$ have the same boundary condition and value based on the inlet turbulence intensity as shown in Table 1 . The $k \overline{v_{2}} \omega$ model has been used for EARSM development.

A plot of the pressure coefficient versus the fraction of axial chord, obtained from all models tested, is shown in Fig. 2 for $\operatorname{Re}_{2 i s}=60,000$. Due to the leading edge acceleration of the flow, there is a peak in the pressure coefficient on the suction side. All the models capture this peak quite well at both Reynolds numbers. Towards the trailing edge of the blade, a plateau is observed in the DNS which is a result of a separation-bubble. Only the LKE and $\mathrm{k} \overline{v_{2}} \omega$ models capture this plateau; more accurately at $\operatorname{Re}_{2 i s}=60,000$ than at $\operatorname{Re}_{2 i s}=100,000$ (not shown here). The other models exhibit no separation at the trailing edge. The $\gamma-\operatorname{Re}_{\theta}$ model, even though expected to, does not capture this separation region. All the models predict the pressure coefficient quite accurately on the pressure side as the flow remains laminar.

Figure 3 depicts the normalized wall shear stress profile on the suction side of the blade at $\operatorname{Re}_{2 i s}=60,000$. There is a smallscale separation bubble near the leading edge, shown by negative $\tau_{w}$, at $\mathrm{x} / \mathrm{C}_{a x} \approx 0.02$ which is larger in the $\mathrm{Re}_{2 i s}=100,000$ case (not shown here) as compared to the $\mathrm{Re}_{2 i s}=60,000$ case. All the models, except the SA model, capture this bubble; more precisely in the $\operatorname{Re}_{2 i s}=60,000$ case than the $\operatorname{Re}_{2 i s}=100,000$ case. The trend of the flow reattachment at $\mathrm{Re}_{2 i s}=60,000$ is predicted quite well by all transition models. At about $\mathrm{x} / \mathrm{C}_{a x} \approx 0.6$, an adverse pressure gradient starts to form due to flow deceleration. There is thus a drop in the wall-shear stress and a laminar separation is formed at $\mathrm{x} / \mathrm{C}_{a x} \approx 0.85$ in the DNS results for both cases; which is not predicted by the $k \omega$-SST, SA or the $\gamma-R e_{\theta}$ models. The LKE and $k \overline{v_{2}} \omega$ models do predict a separation bubble which is longer and thicker than the DNS, and a summary of the lengths of the bubbles is shown in Table 2 .

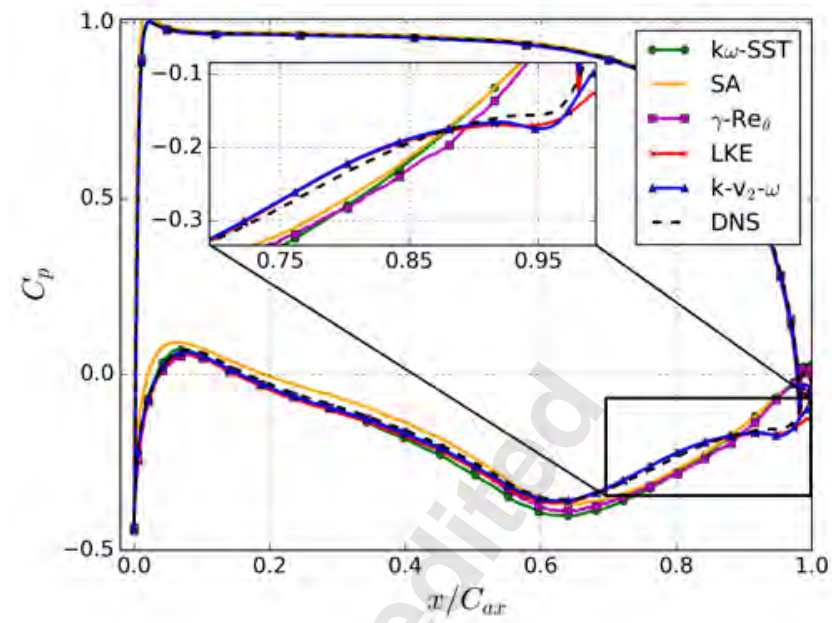

Fig. 2: Pressure coefficient distribution across the blade at $\operatorname{Re}_{2 i s}=60,000$. Inset showed zoomed view of suction side $(0.7 \leq$ $\left.x / C_{a x} \leq 1\right)$

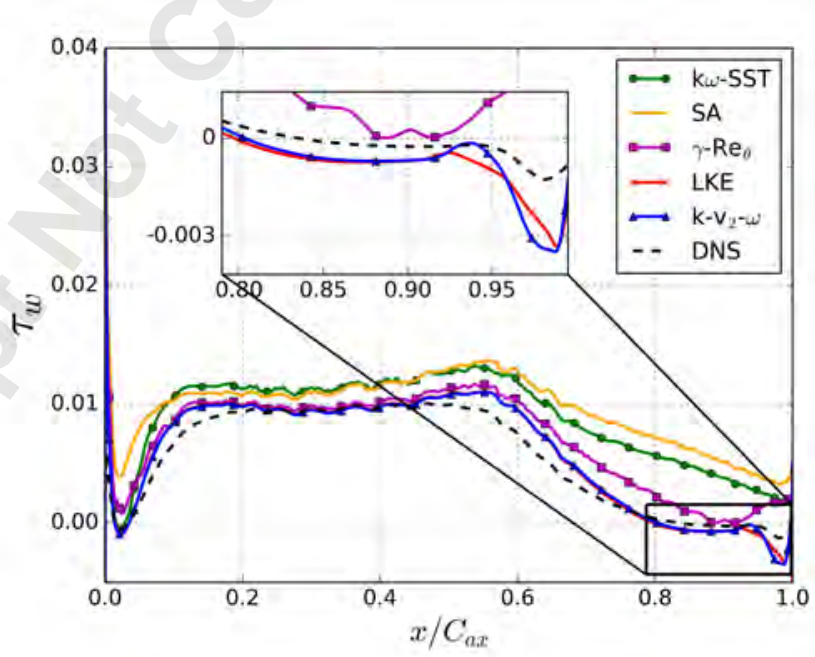

Fig. 3: Wall shear stress on the suction side at $\operatorname{Re}_{2 i s}=60,000$. Inset shows zoomed view of $\left(0.79 \leq x / C_{a x} \leq 1\right)$

Table 2: Length of trailing edge separation bubbles as a fraction of $C_{a x}$

\begin{tabular}{ccc}
\hline $\mathrm{Re}_{2 i s}$ & 60,000 & 100,000 \\
\hline $\mathrm{DNS}$ & 0.17 & 0.13 \\
$k \overline{\nu_{2}} \omega$ & 0.19 & 0.12 \\
$\mathrm{LKE}$ & 0.20 & 0.15 \\
\hline
\end{tabular}

Boundary layer properties such as displacement and momentum thicknesses and shape factor are shown in Fig. 4 for the suction side at $\operatorname{Re}_{2 i s}=60,000$. The momentum thickness predicted by the $k \overline{v_{2}} \omega$ model very closely matches the DNS data. The $k \overline{v_{2}} \omega$ model also outperforms other models in the areas of displacement thickness and shape factor. The most important difference between the $\operatorname{Re}_{2 i s}=60,000$ and $\operatorname{Re}_{2 i s}=100,000$ cases is that the separation bubble reattaches (closed-separation) in the $\operatorname{Re}_{2 i s}=100,000$ case at $\mathrm{x} / \mathrm{C}_{a x} \approx 0.98$, while there is an open- 


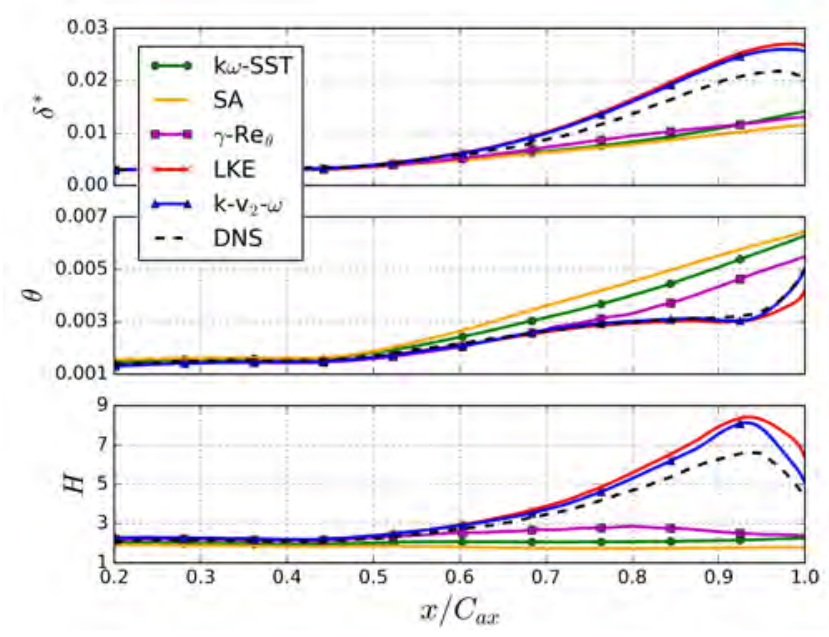

Fig. 4: Displacement thickness, momentum thickness and shape factor on the suction side at $\operatorname{Re}_{2 i s}=60,000$
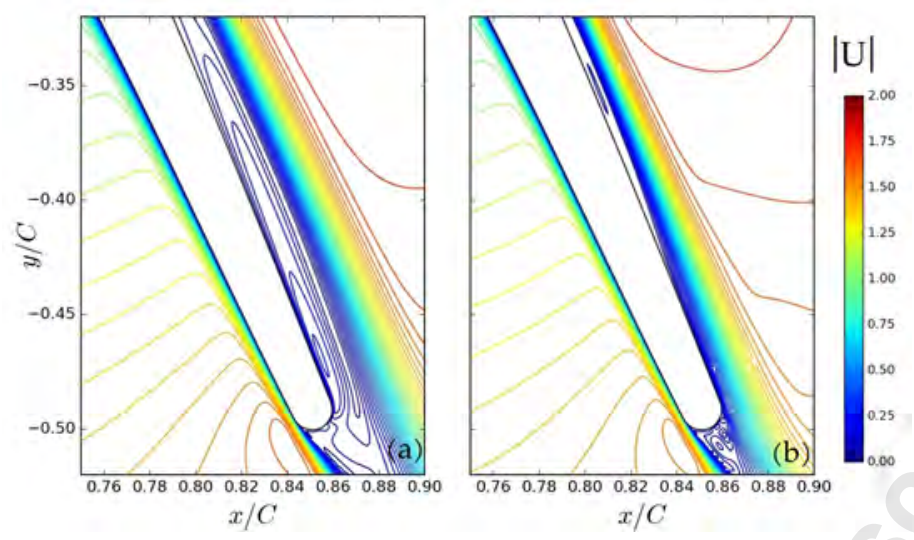

Fig. 5: Magnitude of velocity contours depicting open and closed separation bubbles at (a) $\operatorname{Re}_{2 i s}=60,000$ and (b) $\operatorname{Re}_{2 i s}=100,000$, generated using the $k \overline{v_{2}} \omega$ model

separation in the $\operatorname{Re}_{2 i s}=60,000$ case. Figure 5 displays the open and closed separation bubbles of the two different Reynolds number cases as obtained using the $k \overline{v_{2}} \omega$ model. Both these phenomena are predicted by the LKE and $k \overline{v_{2}} \omega$ models in the respective cases. The separation bubble is thinner at $\operatorname{Re}_{2 i s}=100,000$ as compared to $\operatorname{Re}_{2 i s}=60,000$. These phenomena greatly affect the nature and magnitude of the mixed-out wake loss.

Figure 6 shows the wake loss profiles at $40 \%$ chord downstream of the blade trailing edge for $\mathrm{Re}_{2 i s}=60,000$ and $\operatorname{Re}_{2 i s}=100,000$; plotted against a pitch-wise normalized coordinate, from the suction to the pressure side, defined as $y^{*}=$ $\frac{y-y_{\max }}{y_{\max }-y_{\min }}$, from 0 to 1 . The wake loss is defined below

$$
\Omega=\frac{p_{t, 1}-p_{t, 2}\left(y^{*}\right)}{p_{t, 1}-p_{2}} .
$$

Some of the RANS models ( $k \omega$-SST, SA $\left.\& \gamma-R e_{\theta}\right)$ cannot predict comparable wake loss profiles and either depict very little smearing of the wake $\left(k \omega\right.$-SST $\left.\& \gamma-R e_{\theta}\right)$ or incorrect smearing (SA). This is the expected behavior of non-transitional RANS models as per previous studies [4]. The LKE model produces quite a good prediction of the overall diffusion but fails to capture the peak magnitude, more prominently for $\operatorname{Re}_{2 i s}=100,000$.
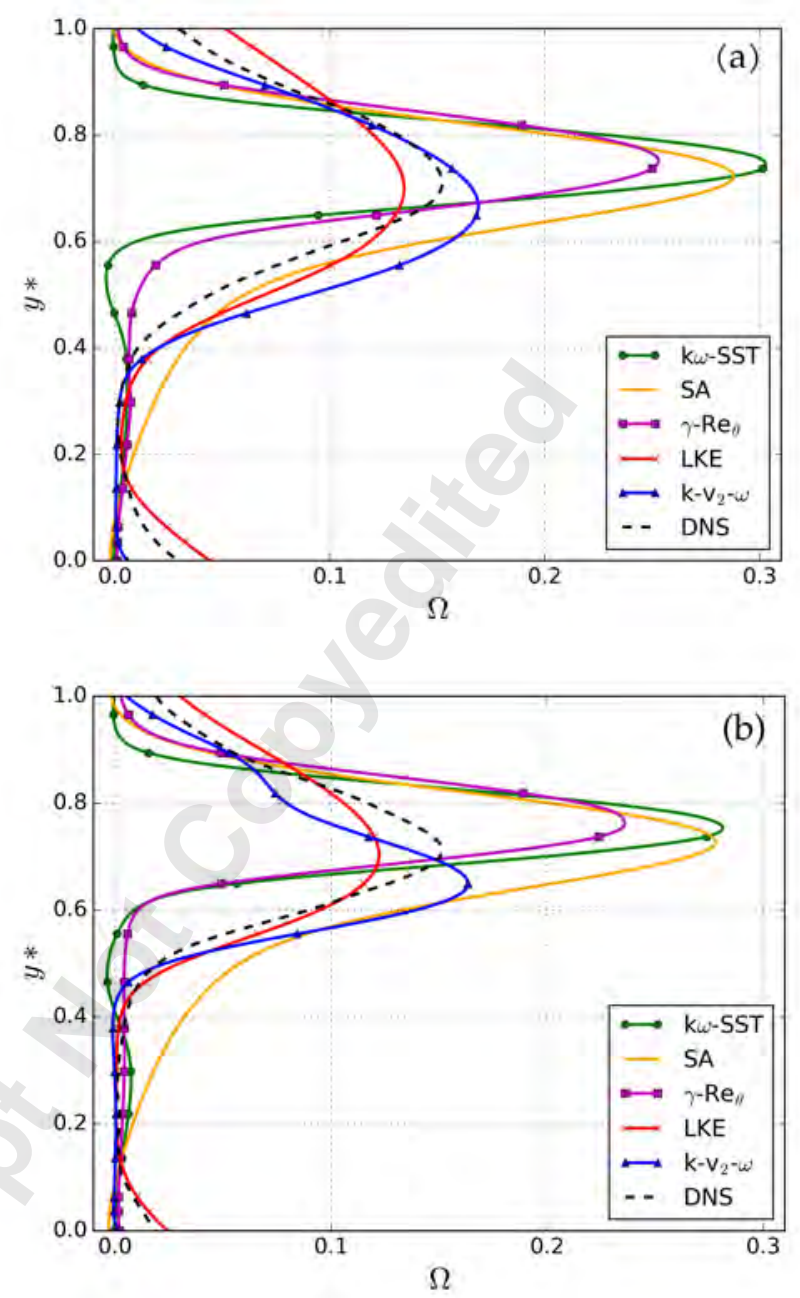

Fig. 6: Wake loss profiles for (a) $\operatorname{Re}_{2 i s}=60,000$ and (b) $\operatorname{Re}_{2 i s}=100,000$, at $40 \%$ chord downstream of the trailing edge

At $\operatorname{Re}_{2 i s}=60,000$, the $k \overline{v_{2}} \omega$ model offers a good comparison of the wake-loss, especially at the peak. This model has an inbuilt cross-diffusion function, similar to the $\mathrm{k} \omega$-SST, and therefore it has a larger peak as compared to the LKE model. However, in the $\operatorname{Re}_{2 i s}=100,000$ case, the $\mathrm{k} \overline{v_{2}} \omega$ model does not capture the location of the peak in the wake loss profile correctly and slightly over-predicts the maximum loss value. The shift in the location can be attributed to the inaccurate prediction of the separated flow coming off the trailing edge and also due to a change of outlet angle; which are better captured by the LKE model.

Out of all the RANS models, the LKE and $k \overline{\nu_{2}} \omega$ model offer the best performance as compared to the DNS in all the four aspects shown above. One of these models has to be selected for EARSM development using GEP. It has been found in this study and in [30] that the LKE model produces non-physical laminar kinetic energy in the wake region - a region that is to be dominated by turbulent flow. This study also found that the $k \overline{v_{2}} \omega$ model offered a more accurate prediction of Reynolds stresses in the wake as compared to the LKE model. Moreover, the $k \overline{v_{2}} \omega$ model [19] was developed in order to address the shortcomings of the LKE model. It has also been shown that the $k \overline{v_{2}} \omega$ model 
offers better boundary layer prediction over the LKE model. Finally, the $\omega$ transport equation of the $k \overline{v_{2}} \omega$ model has a crossdiffusion term which is activated in the wake region to enhance the prediction of wake-mixing. Therefore, the $k \overline{v_{2}} \omega$ model has been chosen for the development of EARSMs to enhance wake mixing and wake-loss prediction for LPTs.

\section{GENE EXPRESSION PROGRAMMING (GEP) \& MODEL DEVELOPMENT}

The EARSM development is carried out with GEP [15, 16], which is a method based on symbolic regression. High-fidelity DNS data [20] for the $\operatorname{Re}_{2 i s}=60,000$ and $\operatorname{Re}_{2 i s}=100,000$ cases has been used for regression. The model development or training process is outlined in Fig. 7. The high-fidelity (DNS) data of interest are the Reynolds stresses, velocity, temperature, TKE and density. This data is used to solve the transport equations for $\overline{v_{2}}$ and $\omega$ of the $k \overline{v_{2}} \omega$ turbulence model using the 'frozen' methodology [31].

With the 'frozen' methodology only the $\overline{\nu_{2}}$ and $\omega$ equations are solved, while keeping pressure, velocity, temperature, density and TKE fixed throughout the entire calculation, set equal to the converged DNS values. A RANS calculation is then performed solving only the $\overline{v_{2}}$ and $\omega$ transport equations in order to obtain the time scale $(1 / \omega)$ most consistent with the DNS data, but based on the RANS equations to be used in subsequent CFD runs. The time scale is used to calculate the normalized tensor basis and scalar invariants (Eq. (3)). The strain-rate $\left(S_{i j}\right)$ and rotation rate $\left(\Omega_{i j}\right)$ tensors are calculated from the DNS quantities as shown in Fig. 7. The Reynolds stress tensor $\left(\tau_{i j}\right)$ obtained from the DNS solution is used to calculated the anisotropy tensor $\left(a_{i j}^{D N S}\right)$. The GEP algorithm then attempts to fit (model regression in Fig. 7) the anisotropy tensor $\left(a_{i j}^{D N S}\right)$ with a linear combination of the tensor basis functions, in order to derive an EARSM $\left(a_{i j}^{R A N S}\right)$. The GEP algorithm achieves this by minimizing the cost function (a form of normalized mean-square error), based on the DNS anisotropy tensor $\left(a_{i j}^{D N S}\right)$

$$
J\left(a_{i j}^{R A N S}\right)=\frac{1}{N} \sum_{n=1}^{N} \sum_{i=1}^{3} \sum_{j \leq i}\left(w_{i j}\left|a_{i j}^{D N S}-a_{i j}^{R A N S}\right|\right)^{2}
$$

where $w_{i j}$ is the weighting function for the individual components of the anisotropy tensor and $N$ is the total number of points in the training region. In this case $w_{11}=w_{12}=w_{22}=w_{33}=0.25$ and $N \approx 2000$ (for the wake region - Fig. 8). The other two components of the anisotropy tensor are zero as it is $2 \mathrm{D}$ flow. After a fixed number of iterations, the GEP returns an anisotropy tensor $\left(a_{i j}^{R A N S}\right)$ as a linearly independent function of the tensor basis (Eq. (2)). The Reynolds stress term (or anisotropy tensor) appears both in the momentum diffusion term in the RANS equation and in the TKE production term. Regressing for this term will lead to improved wake diffusion and TKE production, which will lead to enhanced prediction of wake-mixing.

Selecting the training region (the data used in the calculation of the cost function) is important to capture the relevant flow features for turbomachinery. Since this study focuses on improving the wake mixing, the region of training will be downstream of the blade trailing edge. It has been shown by Weatheritt et.

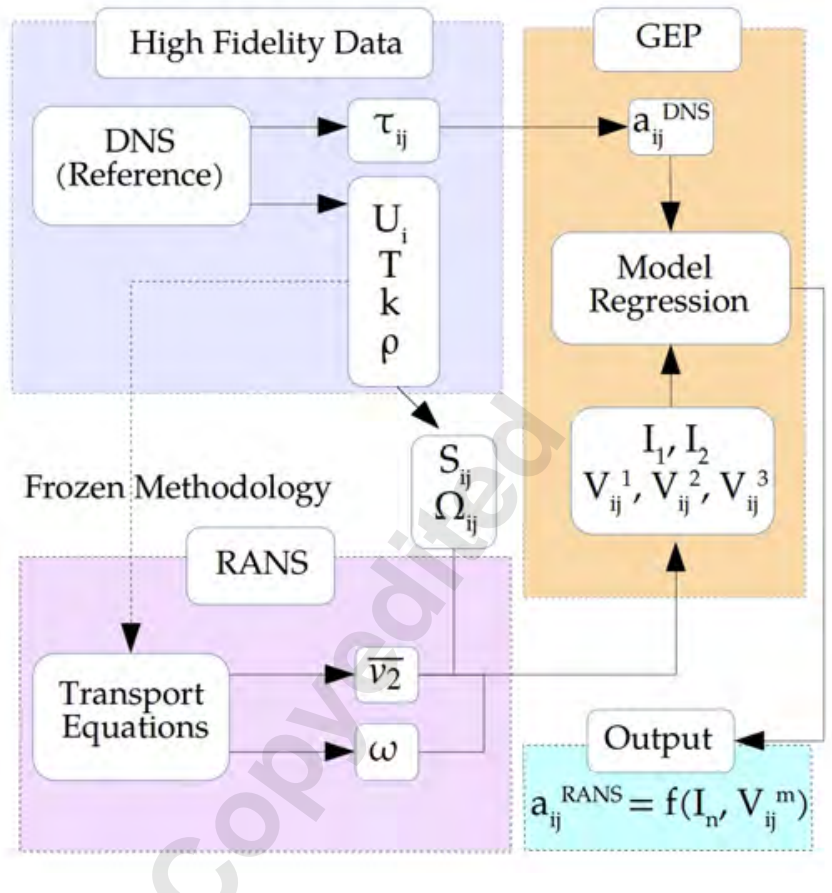

Fig. 7: GEP algorithm flow depicting inputs and output

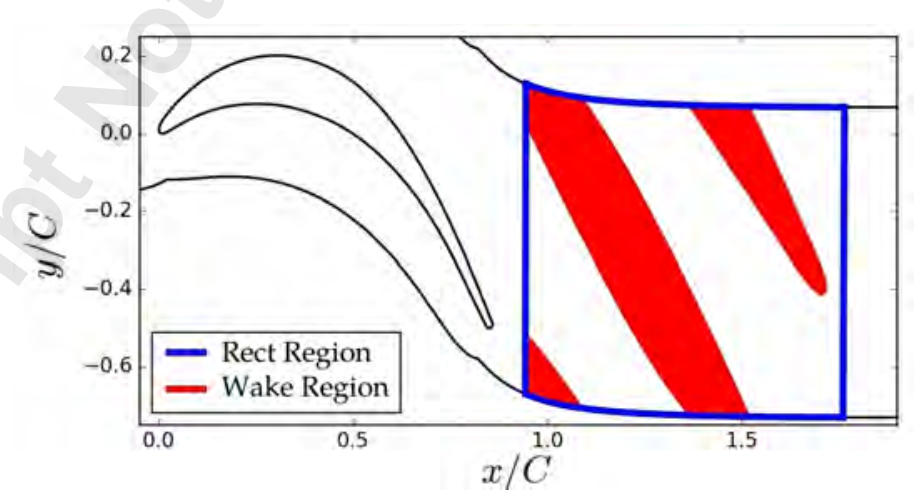

Fig. 8: Training regions for model development

al [18], that if the training region is too close to the trailing edge, i.e. the near-wake region, the nature of the solution is drastically affected. Due to high shear, there is a propagation of error from this region downstream and the trained model produced will not be able to fit the data in the far wake. Therefore, the training region selected begins at a distance $9 \%$ chord downstream of the blade trailing edge. Two possible regions have been considered in addition to the above constraint. One is a rectangular-like (Rect) training region from $x / C=0.95$ to $x / C=1.76$. The other is a region which is meant to capture the data in the wake region (Wake) only and not the passage (Fig. 8). The wake region has been chosen based on a normalized TKE threshold, and hence the width of this region reduces towards the end of the domain. Models trained on the wake region are expected to yield a better prediction of the wake profiles, and are best used with the presence of a wake detection function [32]. A switching function based on the same TKE threshold as the one chosen to define the training region (i.e. Wake region in Fig. 8) was implemented in OpenFOAM to ensure that a model trained on the wake region is activated only in the wake region and not in the passage. Models trained on the rectangular-like region were applied in a 
similar domain during run-time. In the absence of a wake detection function it may be possible that the models trained on a rectangular-like region may offer a good solution as they have been trained to ensure there is no over-fitting to the wake region. Models trained on both these regions are presented below.

\section{DEVELOPED MODELS}

A number of EARSMs, which can modify the Boussinesq approximation (Eq. (2)) for the $k \overline{v_{2}} \omega$ model, have been developed based on the Reynolds number, training region and whether the model has been ensemble-averaged or not (Eqs. 6 - 12). Each complete run of the GEP algorithm produces models which are likely to have different coefficients and slightly different levels of fitness given the non-deterministic nature of this algorithm. To allow for statistical evaluation of model fitness and ensemble averaging, the GEP algorithm was run 50 times and the average of all the models was taken to represent the ensemble averaged model. Table 3 represents the training parameters and the codes to denote each of the trained models. A model which includes data from the wake region of both the 60k and 100k cases has also been developed in order to assess the performance of a model which includes data from more than one DNS set.

Table 3: Model training parameters and codes

\begin{tabular}{|c|c|c|c|}
\hline Reynolds No. & Training Reg. & Ens. Av. & Code \\
\hline 60,000 & Rect & No & $60 \mathrm{k}-$ Rect \\
\hline 100,000 & Rect & No & $100 \mathrm{k}-$ Rect \\
\hline 60,000 & Wake & No & $60 \mathrm{k}-$ Wake \\
\hline 100,000 & Wake & No & $100 \mathrm{k}-$ Wake \\
\hline $60,000 \& 100,000$ & Wake & No & $60 \mathrm{k}-100 \mathrm{k}-W a k e$ \\
\hline 60,000 & Wake & Yes & $60 \mathrm{k}-$ Wake-E \\
\hline 100,000 & Wake & Yes & $100 \mathrm{k}-$ Wake-E \\
\hline
\end{tabular}

60k-Rect:

$$
\begin{aligned}
a_{i j}^{R A N S} & =-\frac{v_{t}}{k} S_{i j}^{\prime}-\left(0.41-2.23 I_{1}\right) V_{i j}^{1}+\left(30.23-2.86 I_{1}\right. \\
& \left.+2.86 I_{2}\right) V_{i j}^{2}+\left(19.44-8 I_{1}+8 I_{2}\right) V_{i j}^{3}
\end{aligned}
$$

100k-Rect:

$$
\begin{aligned}
a_{i j}^{R A N S} & =-\frac{v_{t}}{k} S_{i j}^{\prime}+\left(0.8-2.23 I_{1}+19 I_{2}\right) V_{i j}^{1}+\left(7-32 I_{1}\right. \\
& \left.+22 I_{2}\right) V_{i j}^{2}+\left(11.28-41.84 I_{1}+19.28 I_{2}\right) V_{i j}^{3}
\end{aligned}
$$

60k-Wake:

$$
\begin{aligned}
a_{i j}^{R A N S} & =-\frac{v_{t}}{k} S_{i j}^{\prime}-\left(1.91-I_{1}-14 I_{2}\right) V_{i j}^{1}+\left(21.2-2.36 I_{1}\right. \\
& \left.+16.07 I_{2}\right) V_{i j}^{2}+\left(13.28+I_{2}\right) V_{i j}^{3}
\end{aligned}
$$

100k-Wake:

$$
\begin{aligned}
a_{i j}^{R A N S} & =-\frac{v_{t}}{k} S_{i j}^{\prime}-\left(0.81+2.58 I_{1}-7.16 I_{2}\right) V_{i j}^{1}+(4.41 \\
& \left.-8.21 I_{1}\right) V_{i j}^{2}+\left(16-20 I_{1}+4 I_{2}\right) V_{i j}^{3}
\end{aligned}
$$

60k-100k-Wake:

$$
\begin{aligned}
a_{i j}^{R A N S} & =-\frac{v_{t}}{k} S_{i j}^{\prime}-\left(1.15-2 I_{1}-8 I_{2}\right) V_{i j}^{1}+(6+I 2) V_{i j}^{2} \\
& +\left(24+42 I_{2}\right) V_{i j}^{3}
\end{aligned}
$$

60k-Wake-E:

$$
\begin{aligned}
a_{i j}^{R A N S} & =-\frac{v_{t}}{k} S_{i j}^{\prime}-\left(1.89-2.92 I_{2}\right) V_{i j}^{1}+\left(20.61-6.28 I_{1}\right. \\
& \left.+2.5 I_{2}\right) V_{i j}^{2}+\left(17.49+0.8 I_{1}+0.67 I_{2}\right) V_{i j}^{3}
\end{aligned}
$$

100k-Wake-E:

$$
\begin{aligned}
a_{i j}^{R A N S} & =-\frac{v_{t}}{k} S_{i j}^{\prime}-\left(0.83+1.33 I_{1}-3.26 I_{2}\right) V_{i j}^{1}+(4.26 \\
& \left.-3.24 I_{1}+4.27 I_{2}\right) V_{i j}^{2}+\left(12.12-0.77 I_{1}+1.26 I_{2}\right) V_{i j}^{3}
\end{aligned}
$$
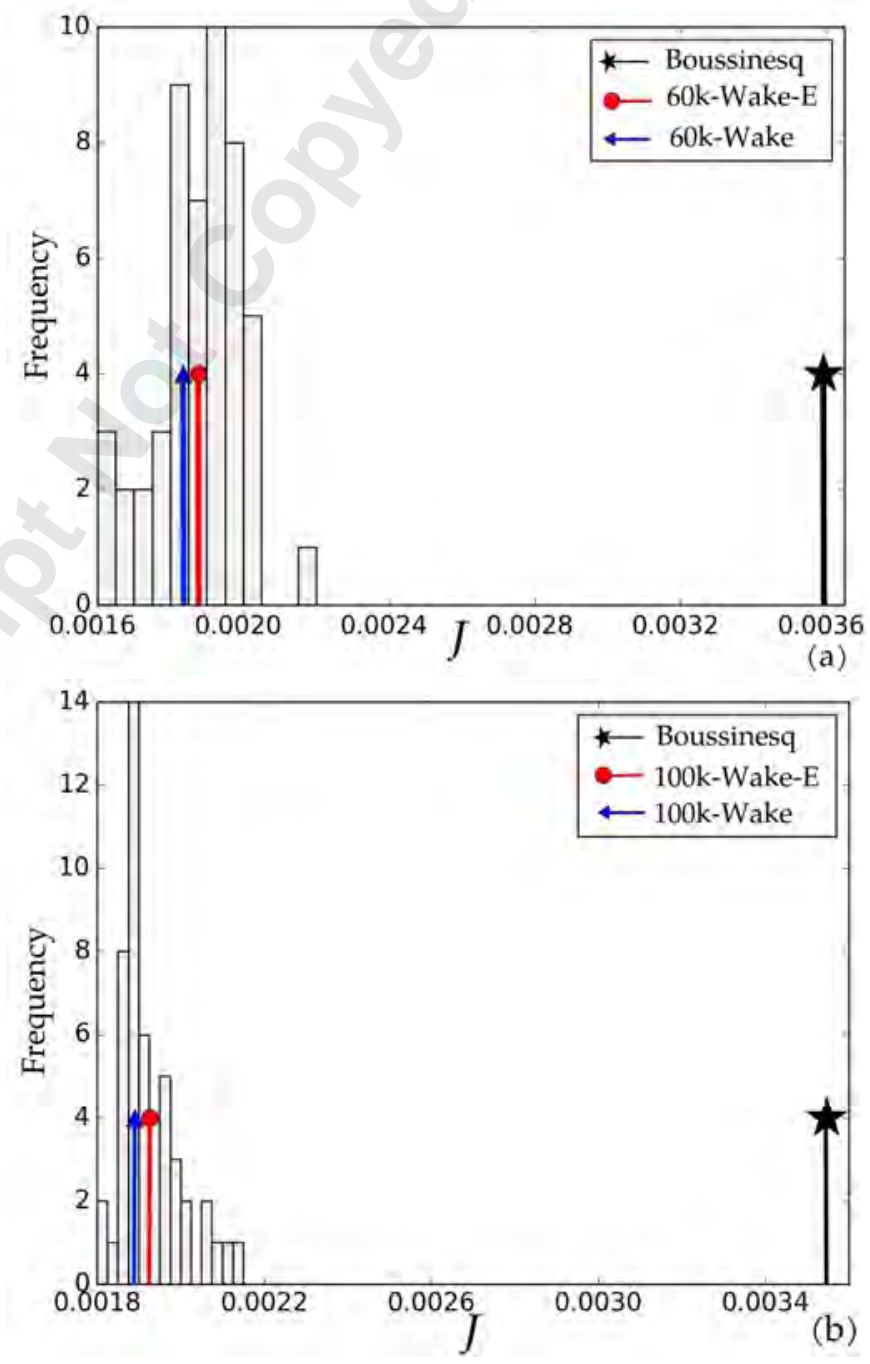

Fig. 9: Histogram of the GEP cost function $(J)$ at (a) $\operatorname{Re}_{2 i s}=60,000$ and (b) $\operatorname{Re}_{2 i s}=100,000$

The values of the normalized mean square error or the GEP cost function $(J)$ for each of the 50 models trained on the $60 \mathrm{k}$ Wake and 100k-Wake regions have been represented in a histogram (Fig. 9). The vertical axis denotes the number of models that have a value of $J$ lying in the respective interval. The values of $J$ derived from the Boussinesq approximation and 60k-Wake, 60k-Wake-E, 100k-Wake \& 100k-Wake-E models have also been symbolically represented (the amplitudes of these quantities have 
no correlation with the frequency) in Fig. 9. It is clear that all the models trained in the $60 \mathrm{k}$-Wake and 100k-Wake regions have yielded an improved fitness (i.e reducing the value of $J$ ) as compared to the linear Boussinesq approximation. This shows that any run of the GEP will return a model which has greatly enhanced the fitness as compared to the linear model. The ensemble averaged results offer an improvement of $47.75 \%$ and $45.56 \%$ in fitness over the linear model for the $60 \mathrm{k}-$ Wake-E and $100 \mathrm{k}$ Wake-E cases respectively. It has been observed that if the normalized mean square error of a model lies within one standard deviation of the normalized mean square error of the ensemble averaged model, there is only a variability of less than $1 \%$ in the wake-loss profiles and Reynolds stress profiles. Therefore, for further analysis, arbitrary models that have a normalized mean square error slightly better than the ensemble averaged cases have been chosen for presentation and the results obtained from the ensemble averaged models have not been further discussed. The fitness of the 60k-Rect, 100k-Rect and 60k-100k-Wake models also lie within one standard deviation of the their respective ensemble averages. It is to be noted that the coefficients of the 60k-Wake and 60k-Wake-E cases differ even though they have a similar fitness. This can be attributed to the non-deterministic nature of the GEP algorithm that can produce two models with entirely different coefficients, yet with the same fitness. It has been found that the majority ( $>90 \%$ ) of error reduction has been brought about by the coefficients of the $V_{i j}^{1}$ and $V_{i j}^{2}$ tensor basis functions. The magnitudes of $I_{1}$ and $I_{2}$ are mostly of the order of 0.01 in the regions of training and $I_{1}$ always is positive whereas $I_{2}$ is negative. Therefore, the coefficients of the tensor basis functions are dominated by the respective constant terms. Hence, the constant coefficients of the $V_{i j}^{1}$ and $V_{i j}^{2}$ bring about the largest influence in error reduction. It can therefore be noted that the constant coefficients of the $V_{i j}^{1}$ and $V_{i j}^{2}$ tensor basis functions for the ensemble averaged cases (60k-Wake-E, 100k-Wake-E) differ by only up to $3 \%$ with respect to the corresponding constant coefficients of the non-ensemble averaged cases (60k-Wake \& 100k-Wake).

The aim of the trained models is to increase the overall diffusion in the region downstream of the blade. It has been observed that the largest improvement in the fitness of the model $(\approx 60 \%)$ has been brought about by the coefficient of the $V_{i j}^{1}$ tensor basis term. This shows that the flow in the wake is strain dominated, and in order to increase the turbulent diffusion and enhance wake-mixing it is anticipated that the coefficient of the $V_{i j}^{1}$ basis function should be negative. All of the models exhibit this trend except for the 100k-Rect model. The models trained on the wake region only focus on the improvement of capturing the wake physics which is strain dominated and not the physics in between the passage which has a higher rotation rate. A positive constant coefficient for the $V_{i j}^{1}$ basis function implies that the diffusion within the wake-passage or Rect region (wake+wakepassage) will be reduced; which is against the expected result. The wake at $\operatorname{Re}_{2 i s}=100,000$ is thinner and exhibits less diffusion or spreading rate than the wake that is formed at $\mathrm{Re}_{2 i s}=60,000$. The wake at $\mathrm{Re}_{2 i s}=60,000$ occupies a larger portion in the Rectregion and therefore, the model that is trained on the $60 \mathrm{k}$-Rect case still exhibits a negative constant coefficient for the $V_{i j}^{1}$ term. In contrast, the Rect region for the $\operatorname{Re}_{2 i s}=100,000$ has a larger contribution from the wake-passage area than the wake region

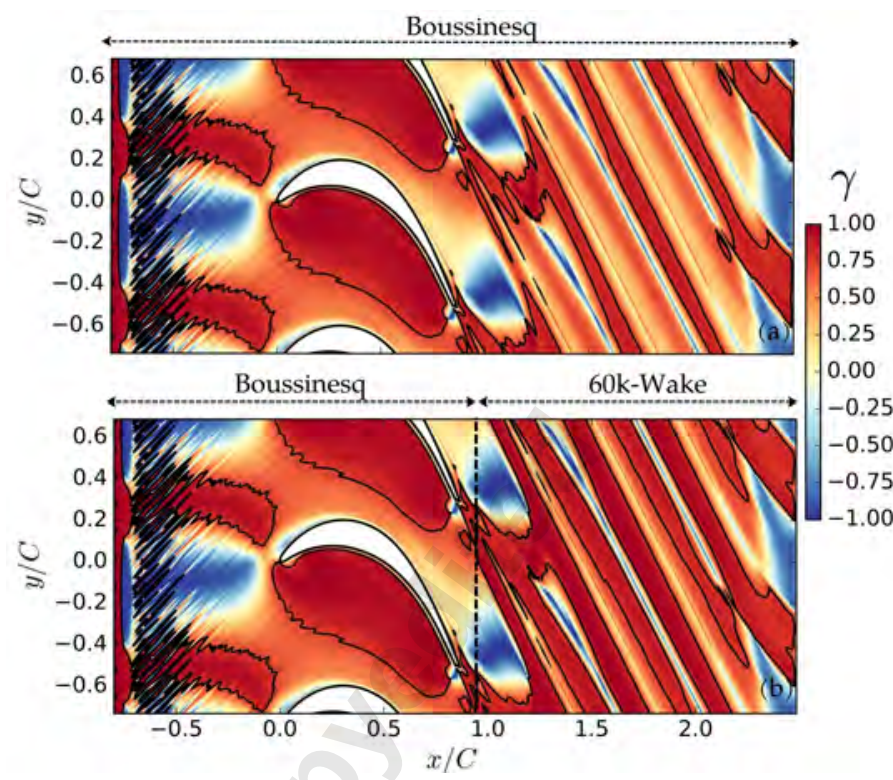

Fig. 10: (a) Alignment of anisotropy and negative strain for the linear Boussinesq approximation. (b) Alignment contour with the linear Boussinesq approximation and 60K-Wake model applied in the regions $x / C<0.95$ AND $x / C \geq 0.95$ respectively, at $\operatorname{Re}_{2 i s}=60,000$. Additional contour at $\gamma=0.75$

which leads to a positive constant coefficient for the $V_{i j}^{1}$ term. This trend is clearly visible from the constant coefficients of the models trained on the wake. The $60 \mathrm{k}$-Wake model has the largest magnitude of the constant term in the $V_{i j}^{1}$ basis function at 1.911, which reduces to 1.15 for the $60 \mathrm{k}-100 \mathrm{k}-$ Wake model and further reduces to 0.81 for the $100 \mathrm{k}-$ Wake model.

\section{A PRIORI RESULTS}

In order to assess the effectiveness of the linear Boussinesq approximation and the stress-strain relationship of trained RANS models in an a priori sense, an extensive linear regression or error-analysis has been conducted, similar to what was conducted by Weatheritt et. al for [18] a high pressure turbine vane, part of which has been presented here.

One of the methods for a priori analysis has been explained below using the 60k-Wake model. Consider the quantity below

$$
\gamma=\frac{a_{i j}^{D N S} a_{i j}^{R A N S}}{\sqrt{a_{m n}^{D N S} a_{n m}^{D N S} a_{p q}^{R A N S} a_{q p}^{R A N S}}}
$$

which is known as alignment $(-1 \leq \gamma \leq 1)$. It measures the orthogonality of the DNS and modeled anisotropy tensors. In order to determine the alignment when the Boussinesq approximation is used, $a_{i j}^{R A N S}$ is to be replaced with the negative strain $\left(-S_{i j}\right)$. $\gamma$ is a useful indicator of the validity of the linear stress-strain relationship [7]. Values close to one imply strong validity of the linear Boussinesq approximation. Values close to minus one imply that Eq. (1) predicts the anisotropy oriented in the wrong direction; while values approximately zero indicate there is zero correlation between the linear model and actual anisotropy (complete orthogonality). Figure 10 (a) shows $\gamma$ for $\operatorname{Re}_{2 i s}=60,000$ with 
an additional contour at $\gamma=0.75$. In Fig. 10(a) the Boussinesq approximation performs well on the pressure side as the flow is laminar and there are no large changes in the velocity gradients. However, the flow is either orthogonal or negatively aligned in the boundary layers as the linear-eddy viscosity hypothesis breaks down in this region. Most of the near wake and far wake are positively aligned, although only a small portion of the wake has a region with $\gamma \geq 0.75$; which shows there is immense scope for improvement with trained RANS models.

Figure 10(b) depicts the alignment between the $a_{i j}^{D N S} \&$ $a_{i j}^{R A N S}$ anisotropy tensors from the 60k-Wake model (Eq. 8). The trained model has been applied in the far-wake region $(x / C \geq$ $0.95,9 \%$ chord downstream), and the Boussinesq approximation is still being used upstream of $x / C=0.95$. The training process has successfully improved the alignment in the far-wake by increasing the area of the wake which has $\gamma \geq 0.75$. This shows that the trained models, in the context of turbomachinery flows, have to be applied in the region which they were regressed for, in order to improve the alignment. The alignment in the wakepassage cannot be significantly improved with a model trained on the wake region due to the different nature of the flow between the two regions. There are a number of other methods such as mean square error analysis to assess the model improvement in an a priori manner, and such analyses have been conducted extensively in the past. However, such a priori metrics do not always offer a reliable prediction of actual CFD performance. Therefore, the next section will discuss the implementation of these trained models into CFD simulations.

\section{IMPLEMENTATION OF DEVELOPED MODELS INTO CFD SIMULATIONS}

In order to assess the improvement in the wake mixing resulting from the trained models (Eqs. 6 - 12), they have been implemented as 2D-URANS simulations for LPTs. Five of these models (excluding the ensemble-averaged models) have been applied from $9 \%$ chord downstream of the trailing edge in conjunction with the $k \overline{v_{2}} \omega$ model for each of the simulations with $\operatorname{Re}_{2 i s}=60,000$ and $\operatorname{Re}_{2 i s}=100,000$. This not only will highlight the performance of the trained models on the training regions and Reynolds numbers that they were developed on, but also will assess their capability of generalizing different flow conditions (in this case open and closed separations on the LPT suction side); which will be a measure of the robustness of the developed models. The baseline case results have been generated using the $k \overline{v_{2}} \omega$ model in conjunction with the Boussinesq approximation. The models which have been trained on in the wake region are active only in the wake region, while the Boussinesq approximation is used for the flow between the wakes.

A direct outcome of the model development process is the improvement in the anisotropy or Reynolds stress prediction in the region $9 \%$ chord downstream of the trailing edge and beyond. Since the physics of the wake is governed by shear-induced turbulent mixing, the most important component to observe an improvement in is $\tau_{x y}$, which is shown in Fig. 11 for both Reynolds numbers at a distance of $40 \%$ chord downstream of the trailing edge (see Fig. 1). At $\operatorname{Re}_{2 i s}=60,000$, the amplitude of the positive peak shear stress in the baseline case is $90 \%$ less than the DNS. The 60k-Wake and 60-Rect models reduce this error to approxi-
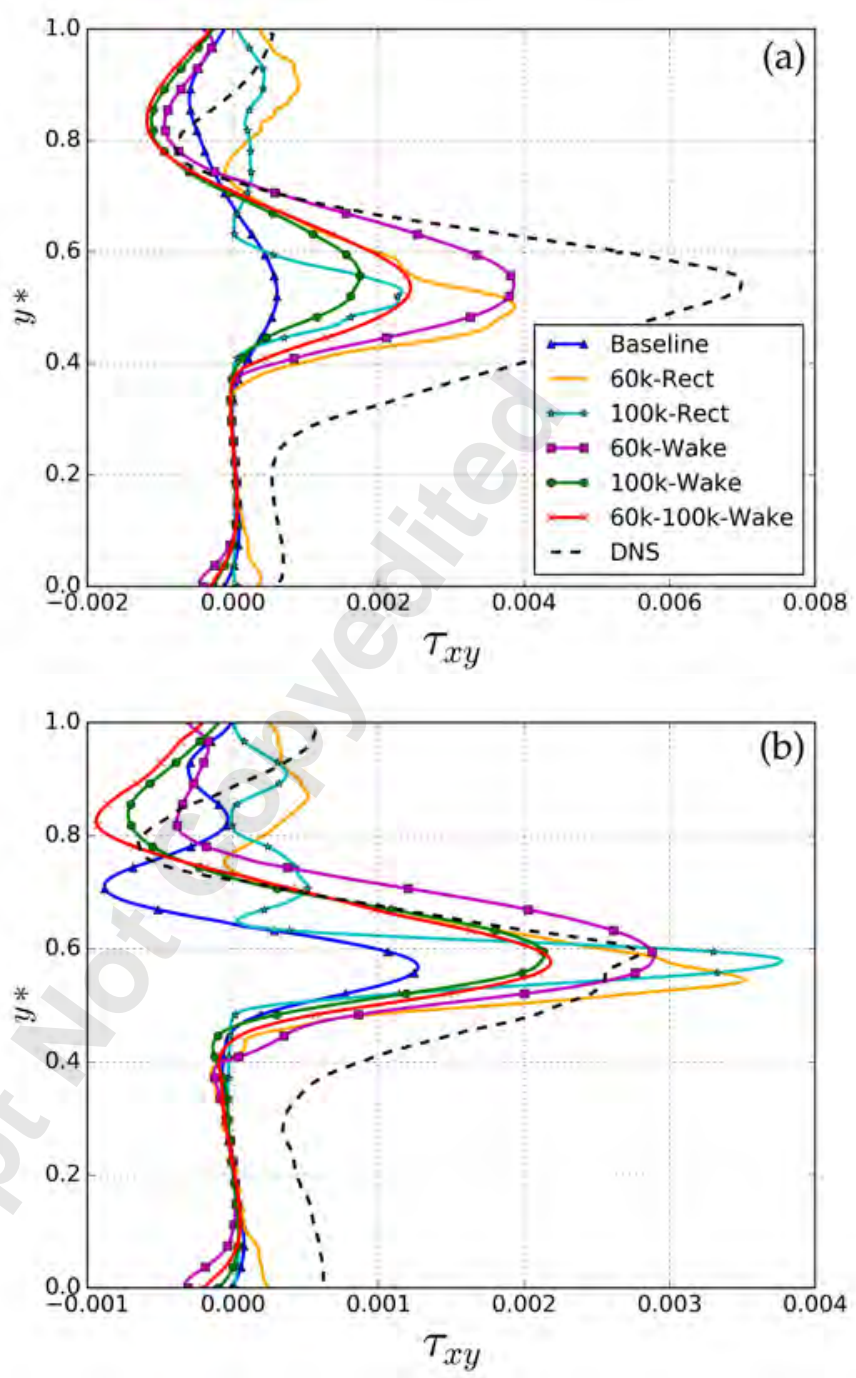

Fig. 11: Shear component of Reynolds stress at $40 \%$ chord downstream for (a) $\operatorname{Re}_{2 i s}=60,000$ and (b) $\operatorname{Re}_{2 i s}=100,000$

mately $45 \%$. The $100 \mathrm{k}-$ Wake and 100k-Rect models also reduce the error in the amplitude of peak stress to $75 \%$ and $68 \%$ respectively. The 60k-100k-Wake model sits in between the stress predictions of the 60k-Wake and 100k-Wake models and reduces the error to $64 \%$. At $\operatorname{Re}_{2 i s}=100,000$, the models trained on the wake region predict the peak and overall-spreading of the shear stress profile better than the ones trained on the rectangular region. The 60k-Wake model produces a higher $\tau_{x y}$ peak value than the other models trained on the wake region due to having a larger constant coefficient in the $-V_{i j}^{1}$ term. In the region from $0 \leq y^{*} \leq 0.4$ all of the models predict an almost vanishing value of $\tau_{x y}$ whereas the DNS predicts a positive stress. This can be explained by the fact that in the case of the RANS simulations, the freestream turbulence is not transported into the wake passage, while it is captured by the DNS.

The anisotropy tensor also appears in the TKE production term. Figure 12 represents the TKE production at $40 \%$ chord downstream of the trailing edge for the two Reynolds numbers. At $\operatorname{Re}_{2 i s}=60,000$, the amplitude of the peak TKE production for the baseline case is $70 \%$ less than the DNS. The models trained 

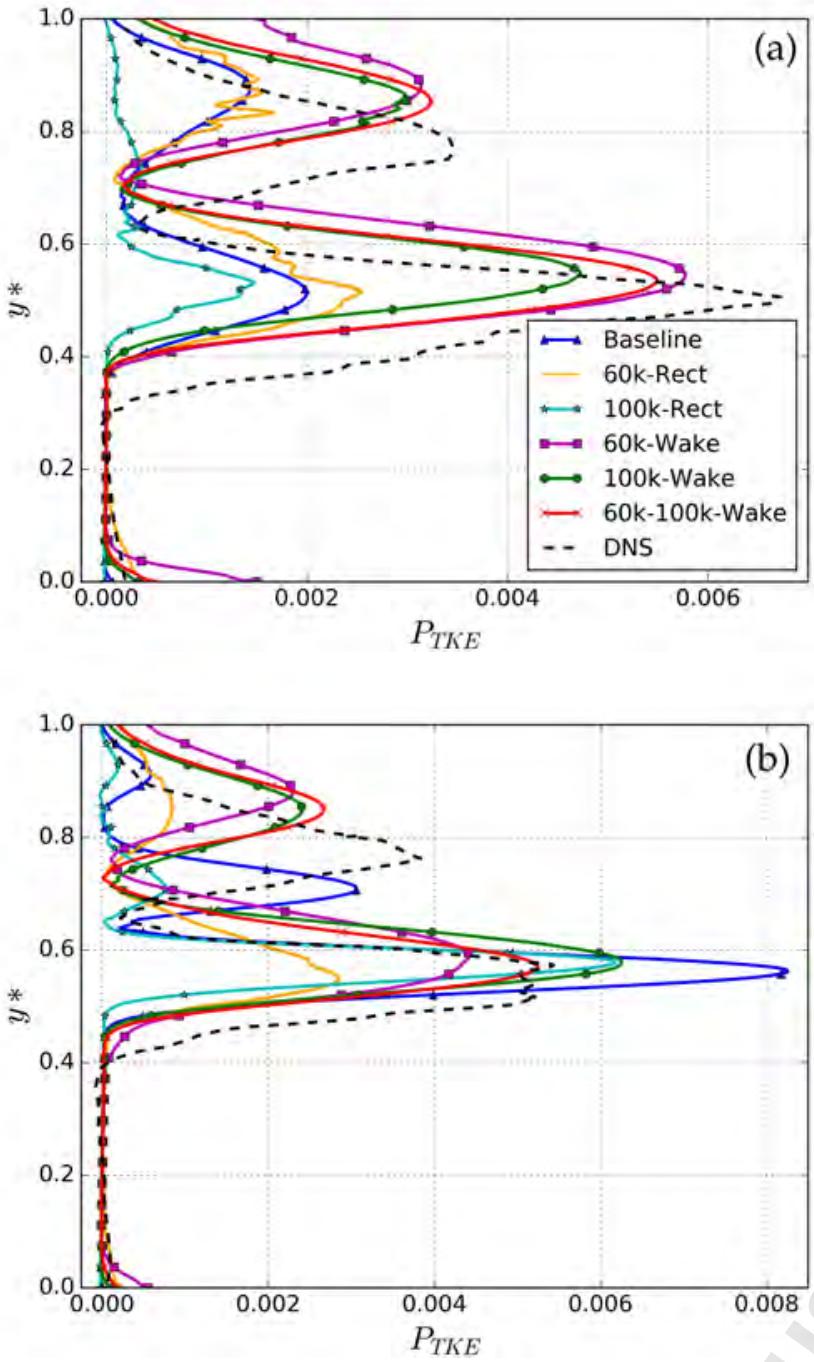

Fig. 12: Production of TKE at $40 \%$ chord downstream for (a) $\operatorname{Re}_{2 i s}=60,000$ and (b) $\operatorname{Re}_{2 i s}=100,000$

on the wake region reduce this error to a range of $12 \%-30 \%$; whereas the models trained on the Rect region bring about no significant change or even reduce the TKE production $(100 \mathrm{k}$ Rect). Even though the models were not regressed for TKE production, some of the models have brought about significant improvements in the TKE production. At $\operatorname{Re}_{2 i s}=100,000$, due to a thinner wake and higher mean velocity gradients as compared to $\mathrm{Re}_{2 i s}=60,000$, there are higher mean shear and Reynold stresses. This causes the baseline RANS model to overpredict the TKE production at its peak $\left(\approx y^{*}=0.55\right)$. The models trained on the wake region seem to have reduced the peak TKE production which shows that the GEP algorithm is able to adapt itself (increase or decrease) to the desired quantity. The TKE profiles from RANS simulations are offset by a small amount as compared to the DNS. It has been observed that although the normal Reynolds stress components have shown improvements in the magnitude of the peak values over the baseline case at both Reynolds numbers, the peak locations are slightly shifted in the pitchwise direction which results in profiles that have a constant offset. Nevertheless, as the flow is shear dominated, this pitch-
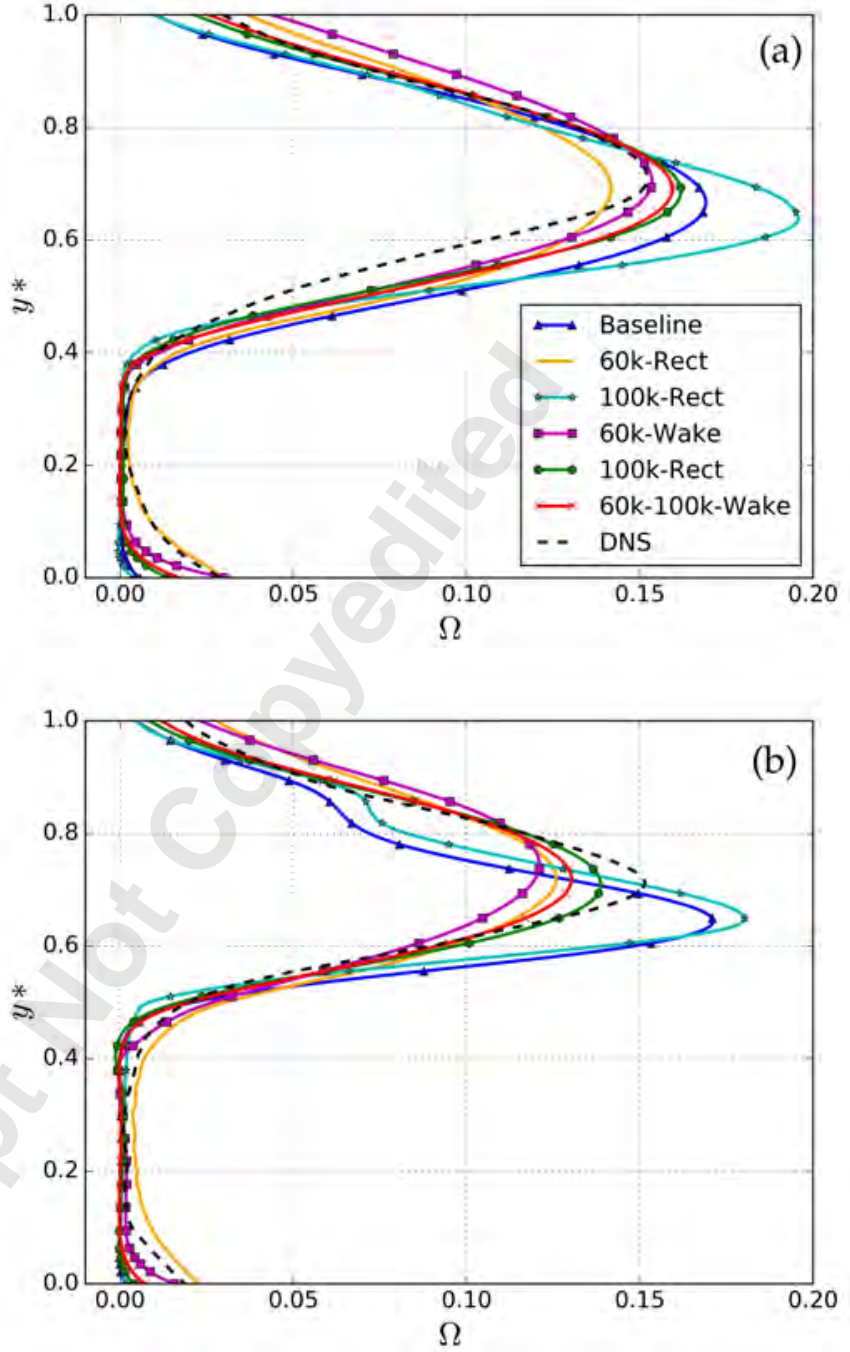

Fig. 13: Wake loss profiles at $40 \%$ chord downstream for (a) $\operatorname{Re}_{2 i s}=60,000$ and (b) $\operatorname{Re}_{2 i s}=100,000$

wise shift in the peaks of the normal Reynolds stress components does not significantly affect the mean flow profile predictions.

Table 4: Mixed-out loss

\begin{tabular}{ccc}
\hline Model & $\mathrm{Re}_{2 i s}=60,000$ & $\mathrm{Re}_{2 i s}=100,000$ \\
\hline DNS & $\mathbf{0 . 0 5 6 1}$ & $\mathbf{0 . 0 4 5 8}$ \\
& \% Error & \\
\hline Baseline & 11.89 & -6.79 \\
60k-Rect & 8.68 & 3.47 \\
100k-Rect & 12.12 & -3.38 \\
60k-Wake & 9.48 & -5.52 \\
100k-Wake & 5.03 & -7.14 \\
60k-100k-Wake & 6.29 & -9.39 \\
\hline
\end{tabular}


It is not only important to improve the stress predictions but also translate that to the improvement in the mean flow quantities, which are ultimately of interest for blade designers. Most of the trained models have enhanced the prediction of pressure and velocity profiles as compared to the baselines cases at both Reynolds numbers, which is reflected in the wake loss profiles (Fig. 13) at $40 \%$ chord downstream. At $\operatorname{Re}_{2 i s}=60,000$, the $60 \mathrm{k}-$ Wake model offers the best performance at the peak. The prediction of the outlet angle has also been improved as the $y^{*}$ location of the peak matches the DNS. Outside of the wake region, for which it was not trained and a linear model was used, there is scope for improvement, especially between $0.1 \leq y^{*} \leq 0.4$. The 60k-Rect model improves the prediction of the wake loss profile to a good degree in regions other than the wake. The model trained on the 100k-Wake and 60k-100k-Wake also perform better than the baseline case in all parts of the wake-loss profile. The 100k-Rect model, due to a positive constant coefficient in the $V_{i j}^{1}$ tensor basis function reduces the diffusion and the result is a narrower wake loss profile, which worsens the prediction of the wake loss profile in all areas over the baseline case. At $\operatorname{Re}_{2 i s}=100,000$, all the trained models, except the 100k-Rect model improve the overall diffusion (shape of profile) and outlet angle prediction. The $100 \mathrm{k}$-Wake model offers the best prediction of the wake loss profile while the $60 \mathrm{k}-100 \mathrm{k}$-Wake model offers an intermediate prediction between the 60k-Wake and 100kWake models. The 60k-Wake model appears to create higher levels of diffusion over other models due to it having being trained on a thicker wake. It is also important to note that there are similar improvements in the $\tau_{x y}$, TKE production, wake loss profiles and thus overall wake-mixing prediction at all locations between $10 \% \mathrm{C}$ to $60 \% \mathrm{C}$ downstream of the blade trailing edge.

Table 4 shows the mixed out loss $\left(\Omega^{M}\right)$ for the DNS defined as $\Omega^{M}=\left(p_{t, 1}^{M}-p_{t, 2}^{M}\right) /\left(p_{t, 1}^{M}-p_{2}^{M}\right)$. The table also shows the percentage error of mixed out loss obtained from the RANS simulations with respect to the DNS. Due to an open separation there is greater mixed out loss at $\operatorname{Re}_{2 i s}=60,000$. At $\operatorname{Re}_{2 i s}=60,000$, all the models except the 100k-Rect model, improve the integral mixed-out loss prediction over the baseline. This follows the trend shown in Fig. 13(a). At $\operatorname{Re}_{2 i s}=100,000$, the models that show the greatest improvements in the wake-loss profiles (Fig. 13(b)), apparently show the highest absolute errors in the mixedout loss values and show greater error as compared to the baseline case. However, the baseline case and the 100k-Rect model (which gives the smallest absolute percentage error as compared to the DNS) do not show a correct wake loss profile shape. Therefore, Fig. 13(b) has to be assessed in conjunction with Table 4 in order to draw a conclusion about their overall performance.

Another parameter to quantify the improvement of wake mixing is the wake maturity, which is the difference between the mixed-out loss and the mass-averaged losses $\left(\Delta \Omega=\Omega^{M}-\right.$ $\left.\Omega^{\text {mass }}\right)$. The wake maturity, normalized with the mixed out loss $\left(\Omega^{M}\right)$, for the baseline case, trained models and the DNS is shown at various locations downstream of the trailing edge for the two Reynolds numbers in Fig. 14, starting from $10 \%$ chord $(x / C=$ $0.96)$ to $60 \%$ chord $(x / C=1.46)$ encompassing the model training region. If a wake were perfectly mixed out, the difference between the mixed out and mass-averaged losses would be zero and it is at a maximum immediately downstream of the trailing edge, where the mixed-out loss remains unchanged but the mass-
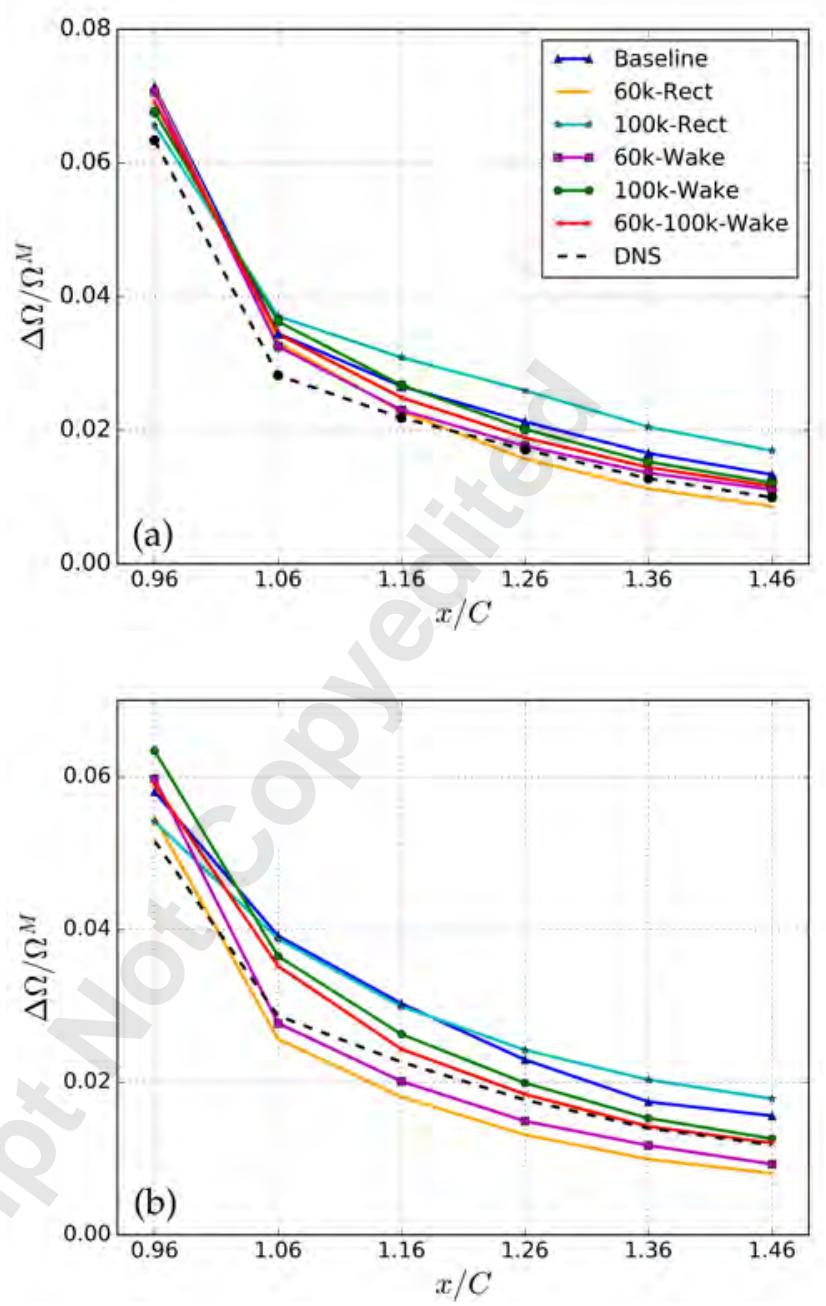

Fig. 14: Non-dimensionalized wake maturity for (a) $\operatorname{Re}_{2 i s}=60,000$ and (b) $\operatorname{Re}_{2 i s}=100,000$

averaged loss is minimum. The wake maturity evolves from large values, in the proximity of the trailing edge, and levels off towards zero further downstream. At both the Reynolds number all the models, except the 100k-Rect model improve the wake mixing beyond a downstream distance of $20 \%$ chord as compared to the baseline case. For the models trained on the wake at both Reynolds numbers, the wake maturity is closer to the DNS as one moves further downstream. An improved wake-maturity prediction also has important implications for the wake which is to be incident on the next stator-blade row. It minimizes the error that is being propagated downstream and thus improves the LPT efficiency.

The models that were trained on a particular Reynolds number and region performed the best when they were used for a RANS calculation at the same Reynolds number and region (e.g. the 60k-Wake model applied in the wake region performed the best at $\operatorname{Re}_{2 i s}=60,000$, as did the $100 \mathrm{k}$-Wake model in the $\operatorname{Re}_{2 i s}=100,000$ case). This shows that the training accuracy of these models is higher than the test accuracy (e.g. models are tested at different conditions than they were developed for). Even though the above holds at this stage of EARSM development for 
LPTs, it is important to note that this is not a simple regression problem. The models which have been trained on high-fidelity data-sets are inserted into partial differential equations, which in turn leads to improved results. Some of the models developed here have performed well even for flow conditions quite different from the training case (i.e. open vs closed separation on the blade) and this shows that some of these models are quite robust and possibly can be applied to different flows, with due consideration. It has also been observed that there is a negligible change in the computation time of the trained models as compared to the baseline case, which demonstrates that it is possible to get significantly improved RANS results at hardly any additional computational cost. There are also no numerical stability issues with the trained models.

\section{CONCLUSIONS}

In this study five turbulence models were applied to the T106A LPT configuration at $\mathrm{M}_{2}=0.4$ and $\mathrm{Re}_{2 i s}=60,000$ and 100,000. To our knowledge, it was the first use of the $k \overline{\nu_{2}} \omega$ model to LPT flows and it captured the LPT suction side separation and offered the best overall prediction of boundary layer flows of all models assessed. However, it was found that there was scope for improvement of the wake-loss profile prediction with the $k \overline{v_{2}} \omega$ model. A priori analysis showed that use of the linear Boussinesq stress-strain relation is one of the significant contributors for inaccurate prediction of the wake loss-profiles. A machine-learning based technique, GEP, was therefore applied to DNS data to develop models that specifically enhance the wake-mixing and wake-loss profiles. The result of the GEP algorithm was a series of non-linear closures (EARSMs) whose performance is found to depend on a number of important factors such as cost functions, training region and ensemble-averaging. The largest contribution to the reduction in normalized mean square error is brought about by the $V_{i j}^{1}$ tensor basis, and thus analyzing its coefficient gave insights into trends of diffusion prediction. The trained models were successfully implemented into OpenFOAM using the $k \overline{v_{2}} \omega$ model as the baseline RANS model at hardly any additional computational cost and with no numerical stability issues. Some of these trained models have significantly improved the $\tau_{x y}$ profiles, which is the dominant Reynolds stress component in the wake. Slight improvements in the normal components of the Reynolds stress were also observed. Even though the TKE production data from the DNS was not used for model development, the production profiles also improved significantly. Most importantly, the improvement in the prediction of the anisotropy tensor translated to improved mean flow quantities. This resulted in enhanced wake-loss profiles and wake maturity predictions, both of which are important parameters for blade designers. The training region is an important parameter for model development and it was found that the models trained on the wake region offer better performance than the ones trained on a region which encompasses the wake and wake passage. Some of the trained models show ability to generalize different kinds of wake flows (wakes being generated from open and closed separation bubbles on the LPT suction side) which is an indication of the robustness of these models. On the whole, the wake-mixing prediction has been enhanced with the CFD (a posteriori) implementation of certain trained models, which demonstrates that GEP for turbulence modeling is a promising avenue for RANS model development for turbomachinery applications.

It is also possible to sensitize models to flow regions with strong pressure gradients, such as along the blade or in the separation bubble area. However, the models trained there will have different coefficients for the tensor basis functions as compared to the ones developed in this paper. This work serves as a building block for more practical scenarios for LPTs; where incoming wakes are considered [33]. Since this methodology has been shown to bring about improvement in prediction of wakes downstream of the trailing edge, there is a very high possibility that the accurate prediction of incoming wakes and their evolution through the turbine passage can be improved to a good degree with the development of EARSMs based on the GEP algorithm.

\section{ACKNOWLEDGMENTS}

This work was supported by resources provided by the Pawsey Supercomputing Centre with funding from the Australian Government and the Government of Western Australia. The support by the Australian Government Research Training Program Scholarship is acknowledged. The University of Melbourne authors also acknowledge the financial support and the permission to publish by General Electric.

\section{NOMENCLATURE}

$a_{i j} \quad$ Normalized anisotropy tensor

C Blade chord length

$H$ Shape factor

$I_{k} \quad$ Scalar invariant

$J \quad$ GEP cost function

$k \quad$ Turbulent kinetic energy

Ma Mach number

$p \quad$ Pressure

$P \quad$ TKE production

Re Reynolds number

$S_{i j} \quad$ Strain rate: $\frac{1}{2}\left(\partial_{x_{j}} U_{i}+\partial_{x_{i}} U_{j}\right)$

$S_{i j}^{\prime} \quad$ Deviatoric component of strain rate: $S_{i j}-\frac{1}{3} \delta_{i j} S_{k k}$

$T$ Temperature

$U_{i} \quad$ Velocities

$\overline{v_{2}} \quad$ Wall-normal turbulent velocity fluctuations

$V_{i j}^{k} \quad$ Tensor basis function

$y^{*} \quad$ Non-dimensional pitchwise coordinate

$\gamma \quad$ Tensor alignment

$\delta \quad$ Displacement thickness

$\theta \quad$ Momentum thickness

$\mu_{t} \quad$ Eddy viscosity

$\tau \quad$ Turbulent time scale $(1 / \omega)$

$\tau_{i j} \quad$ Reynolds stress tensor: $\rho \overline{u_{i}^{\prime} u_{j}^{\prime}}$

$\omega$ Specific dissipation rate

$\Omega \quad$ Loss coefficient

$\Omega_{i j} \quad$ Rotation rate tensor: $\frac{1}{2}\left(\partial_{x_{j}} U_{i}-\partial_{x_{i}} U_{j}\right)$ 


\section{SUBSCRIPTS / SUPERSCRIPTS}

1 Inlet measurement line

2 Exit measurement line

ax Axial

is Isentropic

$t \quad$ Stagnation quantity

$M \quad$ Mixed-out quantity

\section{ACRONYMS}

$\begin{array}{ll}\text { DNS } & \text { Direct numerical simulation } \\ \text { EARSM } & \text { Explicit algebraic Reynolds stress model } \\ \text { GEP } & \text { Gene expression programming } \\ \text { LKE } & \text { Laminar kinetic energy } \\ \text { LPT } & \text { Low pressure turbine } \\ \text { RANS } & \text { Reynolds averaged Navier-Stokes } \\ \text { SA } & \text { Spalart Allmaras } \\ \text { SST } & \text { Shear stress transport } \\ \text { TKE } & \text { Turbulent kinetic energy }\end{array}$

\section{REFERENCES}

[1] Mayle, R. E., 1991. "The Role of Laminar-Turbulent Transition in Gas Turbine Engines". ASME, Trans. J. Turbomach., 113, pp. 509-537.

[2] Hodson, H. P., and Howell, R. J., 2005. "Bladerow Interactions, Transition, and High-Lift Aerofoils in Low-Pressure Turbines". Annu. Rev. Fluid Mech., 37(1), pp. 71-98.

[3] Stieger, R. D., and Hodson, H. P., 2003. "The Transition Mechanism of Highly-Loaded LP Turbine Blades”. ASME Conf. Proc., 2003(36886), pp. 779-788.

[4] Pacciani, R., Marconcini, M., Arnone, A., and Bertini, F., 2013. "Predicting High-Lift Low-Pressure Turbine Cascades Flow Using Transition-Sensitive Turbulence Closures". J. Turbomach., 136(5), p. 051007.

[5] Keadle, K., and Mcquilling, M., 2013. "Evaluation of RANS Transition Modeling for High Lift LPT Flows at Low Reynolds Number". In Proc. ASME Turbo Expo 2013 Turbine Tech. Conf. Expo., pp. GT2013-95069.

[6] Praisner, T. J., Clark, J. P., Nash, T. C., Rice, M. J., and Grover, E. A., 2006. "Performance Impacts Due to Wake Mixing in Axial Flow Turbomachinery”. In ASME Turbo Expo, no. GT2006-90666.

[7] Schmitt, G., 2007. “About Boussinesq's Turbulent Viscosity Hypothesis: Historical Remarks and a Direct Evaluation of its Validity.". Comptes Rendus Mec. Elsevier Masson, 335(9), pp. 617-627.

[8] Leschziner, M., 2015. Statistical Turbulence Modelling for Fluid Dynamics - Demystified: an Introductory Text for Graduate Engineering Students. World Scientific.

[9] Rodi, W., 1976. "A New Algebraic Relation for Calculating the Reynolds Stresses". In Gesellschaft Angew. Math. und Mech. Work. Paris Fr., Vol. 56.

[10] Gatski, T., and Speziale, C., 1993. "On Explicit Algebraic Stress Models for Complex Turbulent Flows". J. Fluid Mech., 254, pp. 59-78.

[11] Wallin, S., and Johansson, A. V., 2000. "An Explicit Algebraic Reynolds Stress Model for Incompressible and Compressible Turbulent Flows”. J. Fluid Mech., 403, pp. 89-132.

[12] Wang, J.-X., Wu, J.-L., and Xiao, H., 2017. “A Physics Informed Machine Learning Approach for Reconstructing Reynolds Stress Modeling Discrepancies Based on DNS Data". Phys. Rev. Fluids, 2(3), p. 034603.

[13] Duraisamy, K., Zhang, Z. J., and Singh, A. P., 2015. "New Approaches in Turbulence and Transition Modeling Using Data-driven Techniques". 53rd AIAA Aerosp. Sci. Meet., 1284.

[14] Tracey, B. D., Duraisamy, K., and Alonso, J. J., 2015. "A Machine Learning Strategy to Assist Turbulence Model Development". 53rd AIAA Aerosp. Sci. Meet.(January), pp. 1-22.
[15] Ferreira, C., 2001. "Gene Expression Programming: a New Adaptive Algorithm for Solving Problems”. Complex Syst., 13(2), pp. 87-129.

[16] Weatheritt, J., and Sandberg, R. D., 2016. "A Novel Evolutionary Algorithm Applied to Algebraic Modifications of the RANS Stress-Strain Relationship". J. Comput. Phys., 325, pp. 22-37.

[17] Weatheritt, J., and Sandberg, R. D., 2017. "The development of algebraic stress models using a novel evolutionary algorithm". Int. J. Heat Fluid Flow, 68(September), pp. 298-318.

[18] Weatheritt, J., Pichler, R., Sandberg, R. D., Laskowski, G., and Michelassi, V., 2017. "Machine Learning for Turbulence Model Development Using a High Fidelity HPT Cascade Simulation". In ASME Turbo Expo 2017 Turbomach. Tech. Conf. Expo., pp. GT2017-63497.

[19] Lopez, M., and Walters, D. K., 2016. "Prediction of Transitional and Fully Turbulent Flow Using an Alternative to the Laminar Kinetic Energy Approach". J. Turbul., 17(3), pp. 253-273.

[20] Michelassi, V., Chen, L. W., Pichler, R., and Sandberg, R. D., 2015. "Compressible Direct Numerical Simulation of Low Pressure Turbine - Part II: Effect of Inflow Disturbances". J. Turbomach., 137(7), p. 71005.

[21] Pope, S. B., 1975. "A More General Effective-Viscosity Hypothesis". J. Fluid Mech., 72(2), pp. 331-340.

[22] Sandberg, R. D., Michelassi, V., Pichler, R., Chen, L., and Johnstone, R., 2015. "Compressible direct numerical simulation of low-pressure turbinespart I: Methodology”. J. Turbomach., 137(5), p. 51011.

[23] Stadtmüller, P., and Fottner, L., 2001. "A Test Case for the Numerical Investigation of Wake Passing Effects on a Highly Loaded LP Turbine Cascade Blade". Vol. 1 Aircr. Engine; Mar. Turbomachinery; Microturbines Small Turbomach., 1, p. V001T03A015.

[24] Menter, F. R., 1994. "Two-equation eddy-viscosity turbulence models for engineering applications". AIAA J., 32(8), pp. 1598-1605.

[25] Spalart, P. R., and Allmaras, S. R., 1994. "A one-equation turbulence model for aerodynamic flows". La Rech. Aerosp., 1, pp. 5-21.

[26] Langtry, R. B., and Menter, F. R., 2009. "Correlation-based transition modeling for unstructured parallelized computational fluid dynamics codes". AIAA J., 47(12), pp. 2894-2906.

[27] Walters, D. K., and Cokljat, D., 2008. "A Three-Equation Eddy-Viscosity Model for Reynolds-Averaged Navier-Stokes Simulations of Transitional Flow". J. Fluids Eng., 130(12), p. 121401.

[28] Mayle, R., and Schulz, A., 1997. "The Path to Predicting Bypass Transition". J. Turbomachinery. Trans. ASME, 119(3), pp. 405-411.

[29] Dick, E., and Kubacki, S., 2017. "Transition Models for Turbomachinery Boundary Layer Flows: A Review”. Int. J. Turbomachinery, Propuls. Power, 2(2), p. 4.

[30] Lopez, M., and Walters, D. K., 2017. "A Recommended Correction to the kT-kL-omega Transition-Sensitive Eddy-Viscosity Model”. J. Fluids Eng., 139(2), p. 24501.

[31] Parneix, S., Laurence, D., and Durbin, P. A., 1998. "A Procedure for Using DNS Databases”. J. Fluids Eng. Trans. ASME, 120(1), pp. 40-46.

[32] Bode, C., Aufderheide, T., Friedrichs, J., and Kozulovic, D., 2014. "Improved Turbulence and Transition Prediction for Turbomachinery Flows". In ASME 2014 Int. Mech. Eng. Congr. Expo., pp. IMECE2014-36866.

[33] Akolekar, H. D., Sandberg, R. D., Hutchins, N., Michelassi, V., and Laskowski, G., 2018. "Machine-Learnt Turbulence Closures for LPTs with Unsteady Inflow Conditions". In 15th ISUAAAT, Oxford, UK. 


\section{University Library}

\section{- M M N E R VA A gateway to Melbourne's research publications}

Minerva Access is the Institutional Repository of The University of Melbourne

Author/s:

Akolekar, HD;Weatheritt, J;Hutchins, N;Sandberg, RD;Laskowski, G;Michelassi, V

Title:

Development and Use of Machine-Learnt Algebraic Reynolds Stress Models for Enhanced Prediction of Wake Mixing in Low-Pressure Turbines

Date:

2019-04-01

Citation:

Akolekar, H. D., Weatheritt, J., Hutchins, N., Sandberg, R. D., Laskowski, G. \& Michelassi, V. (2019). Development and Use of Machine-Learnt Algebraic Reynolds Stress Models for Enhanced Prediction of Wake Mixing in Low-Pressure Turbines. Journal of Turbomachinery, 141 (4), https://doi.org/10.1115/1.4041753.

Persistent Link:

http://hdl.handle.net/11343/241880 